\title{
Genetic Variability for Downy Mildew Disease Incidence against Virulent Downy Mildew Isolates in Mapping Population of Pearl Millet
}

\author{
Anishetti Saritha ${ }^{1}$, Rakesh K. Srivastava ${ }^{2}$, Chelpuri Durgaraju ${ }^{1}$, Narayan Reddy ${ }^{3}$, \\ Rajan Sharma ${ }^{2}$, Pooja Katiyar ${ }^{2}$ and Kuldeep Singh Dangi ${ }^{{ }^{*}}$ \\ ${ }^{1}$ Department of Genetics and Plant Breeding, College of Agriculture, \\ Rajendranagar, 500 030, Telangana, India \\ ${ }^{2}$ International Crops Research Institute for the Semi-Arid Tropics, \\ Patancheru, 502324, Hyderabad, Telangana, India \\ ${ }^{3}$ Department of Plant Pathology, College of Agriculture, Rajendranagar-500 030, India \\ *Corresponding author
}

A B S T R A C T

\begin{tabular}{|c|}
\hline \\
\hline $\begin{array}{l}\text { Pearl millet, } \\
\text { Downy mildew, } \\
\text { Inoculation, Isolates, } \\
\text { Pathogenicity, } \\
\text { Screening, } \\
\text { Resistance, Virulent. }\end{array}$ \\
\hline Article Info \\
\hline $\begin{array}{l}\text { Accepted: } \\
07 \text { September } 2017 \\
\text { Available Online: } \\
10 \text { October } 2017\end{array}$ \\
\hline
\end{tabular}

Keywords

Pearl millet,

Downy mildew,

Pathogenicity,

Screening,

Resistance, Virulent.

Accepted: Available Online:

\section{Introduction}

Pearl millet (Pennisetum glaucum (L.) R. $\mathrm{Br}$.), is an important cereal crop. It is especially important as a staple food grain and source of feed and fodder for livestock, in hot, dry marginal agricultural production environments of Africa and South Asia that are home to millions of the world's poorest farmers (Rajaram et al., 2013).

Pearl millet, with $8-19 \%$ seed protein content and $56-65 \%$ carbohydrates is nutritionally superior to rice, sorghum and maize. Pearl millet downy mildew is the most destructive disease that is responsible for substantial economic losses. The estimated annual grain yield loss due to downy mildew is approximately 20-40\% (Thakur et al., 2008). The disease is more severe on genetically homogeneous single-cross pearl millet hybrids, which are grown on about $60 \%$ of the total 9.5 million ha in India, than on heterogeneous open-pollinated varieties 
(Thakur et al., 2006). During the 1970s- 80s several downy mildew epidemics occurred in India resulting in considerable yield losses and withdrawal of several hybrids from cultivation (Singh et al., 1987; Thakur, 1999). Currently, over 70 different hybrids are being grown in India (Thakur et al., 2006) and during our recent on-farm survey, some of them have shown downy mildew incidence up to $100 \%$. The on-farm downy mildew surveys in the major pearl millet growing states of India have revealed that several commercial $\mathrm{F}_{1}$ hybrids being grown in different states become susceptible to the disease within 3-5 years (Thakur et al., 2003; Rao et al., 2005; Thakur et al., 2006). Existence of mating types and their frequency greatly contribute towards the development of new recombinants in the pathogen populations (Pushpavathi et al., 2006a). Evolution of hostspecific virulence in pearl millet downy mildew is well documented (Thakur et al., 1992; Sastry et al., 2001; Pushpavathi et al., 2006b). Since management of pearl millet downy mildew largely depends upon host plant resistance, evolution of new virulence(s) in the pathogen population and resistance effective against new pathotypes need to be periodically monitored. Effective and economic control of this disease can be achieved by growing disease resistant varieties and hybrids. So, disease resistance is a major concern in pearl millet improvement programme. In order to develop disease resistant cultivars, it is important to identify the germplasm lines which can be used as parents for the development of mapping populations which invariably useful in identification of QTLs and candidate genes governing resistance to the DM.

\section{Materials and Methods}

The present experiment was carried out with mapping population of 295 RILs develped by crossing of 81B-P13 and AIMP 92901-derivP03 at ICRISAT and two control entries viz.,
7042(S) and IP18292. They were evaluated against three isolates of downy mildew pathogen namely Sg445 (Gujarat), Sg519 (Haryana) and Sg526 (Rajasthan) under greenhouse conditions during Kharif 2013, at ICRISAT, Patancheru. Seed of these accessions were obtained from ICRISAT Genetic Resources.

A wide diversity of populations of this pathogen has been identified from India and samples of these are being maintained at ICRISAT, Patancheru (Thakur and Rao, 1993). The pathogen populations are maintained on plants of highly downy mildew susceptible pearl millet genotypes 7042(S) and $F_{1}$ hybrid NHB 3 , both of which show $>80 \%$ infection under heavy inoculum pressure.

The infected plants are grown in sterilized soil in covered pots in a greenhouse room maintained at slightly above atmospheric pressure to prevent the entry of air-borne spores. Seedlings were inoculated at the two or three leaf stage by spray application of a freshly prepared, chilled suspension of pathogen population. The pots were then covered with polythene bags and incubated at $20^{\circ} \mathrm{C}$ to promote infection. After 12 hours, the bags were removed and the pots of seedlings infected with pathogen population were maintained at $20-25^{\circ} \mathrm{C}$ in plexiglass-covers on benches in the greenhouse.

The pot-grown seedlings were Inoculated from its maintainer host with the sporangial suspension of the isolate were incubated at $20^{\circ} \mathrm{C}$ for $16 \mathrm{~h}$ in the dark and then transferred to a greenhouse under misting for 4-5 days. The seedlings were grown for 25-30 days at $25 \pm 2{ }^{\circ} \mathrm{C}$ under proper care of watering and fertilization to produce good infected foliage, which sporulated profusely and provided a good amount of sporangial inoculum needed for mass inoculation (Thakur et al., 2011). 
The experimental material were sown at uniform depth in the holes (single seed per hole) to achieve uniform emergence of seedlings. Seedlings were grown in $12 \mathrm{~cm}$ diameter plastic pots, filled with a potting mixture of three quarters consisting of soil, sand and farmyard manure in a 3:2:2 by volume and watered them. In the pots uniform holes $(1 \mathrm{~cm})$ were made in saturated soil in the pots using a dibbler stamp (This equipment, which facilitates equidistant sowing of seeds at equal depth in a pot thus reducing variability in emergence time due to sowing depth and distance between seedlings, is a new development). The seeds were covered with a $1-\mathrm{cm}$ layer of potting mixture, irrigated properly and maintained these pots in the greenhouse at $35^{\circ} \mathrm{C}$ till seedling emergence.

The seedlings were counted at the coleoptile to first-leaf stage (3 days after sowing) in each pot and recorded the number on the plastic label (Seedlings in each pot are counted and recorded on the plastic label before inoculation to discount any seedlings emerging after inoculation). The above pots were transferred into the inoculation room on metallic shelves and organized them in rows. Seedlings were inoculated with the sporangial suspension using pneumatic atomizer till runoff ensuring that every seedling has received uniform inoculum. Inoculated seedlings were covered with a polyethylene sheet immediately to provide high humidity required for infection and incubated in the dark at $20^{\circ} \mathrm{C}$ for $16-20 \mathrm{~h}$.

The inoculated seedlings were shifted to greenhouse benches at $25 \pm 2^{\circ} \mathrm{C}$ with misting to provide high humidity ( $>95 \% \mathrm{RH})$ and leaf wetness for disease development for the next 14 days. Infected seedlings in each pot were counted and recorded the number on the same plastic label in the pot on which total seedling counts were recorded before inoculation. The same inoculation procedures were repeated three times [time replications in Completely Randomized Block Designs for each of the three pathogen populations]. Mean downy mildew disease incidence percentage will be calculated for each genotype. Symptoms as distinct chlorosis on leaves and stunted growth of seedlings were recorded. The disease incidence was scored as: highly susceptible - DMI $>80 \%$, susceptible $50 \%$ $80 \%$ DMI, moderately susceptible $25 \%$ $50 \%$ DMI, moderately resistant $10 \%$ $25 \%$ DMI and resistant $<10 \%$ DMI.

\section{Results and Discussion}

Among the 295 RILs under study, 19 lines i.e.5, 19,40, 50,60, 62,73,76,78, 91, 122, 179, 185,186, 205, 225, 238, 274 and 289 were found to be highly susceptible (>80\% DMI) in DM screening against the pathogen isolates from Gujarat (Sg445) and Haryana (Sg519) (Table 1).

Seventeen lines exhibited highly susceptible (>80 \% DMI) in DM screening against the pathogen isolates from Gujarat (Sg445) and Rajasthan (Sg526) (Table 1), numbered those are $68,103,107,120,134,136,145,146$, 155, 164, 166, 174, 203, 233, 248, 278 and 290 among 295 RILs.

Among all the 295 Recombinant Inbred Lines, there are no RILs found to be susceptible against all the three pathogen isolates.

Only thirteen lines shown resistance against all the three isolates, those are $22,42,53,61$, 69, 74, 75, 189, 228, 230, 237, 281 and 291.

Different populations exhibited different levels of DM incidence with three isolates of the pathogen Sclerospora graminicola under investigation. 
Summary revealed that the pathogen population of $S$. graminicola from Gujarat (Sg445) caused the greatest disease incidence $(62.64 \%)$ followed by the pathogen populations from Haryana (Sg519) and Rajasthan (Sg526). A very high heritability estimates for DM1 was observed for screens against $\mathrm{Sg} 526$, followed by $\mathrm{Sg} 519$ and $\mathrm{Sg} 445$.

Table.1 The Mean downy mildew incidence percentage (DMI \%) data from 295 RILs, parents and control entries based on cross 81B-P13 and AIMP 92901-deriv-P03 against three isolates of pathogen Sclerospora graminicola

\begin{tabular}{|c|c|c|c|}
\hline \multirow{2}{*}{ S. No. } & \multicolumn{3}{|c|}{ DMI\% } \\
\hline & Sg445 & Sg519 & Sg526 \\
\hline 1 & 96.00 & 0.00 & 28.15 \\
\hline 2 & 30.83 & 5.60 & 0.00 \\
\hline 3 & 85.50 & 11.00 & 46.10 \\
\hline 4 & 49.13 & 27.67 & 5.95 \\
\hline 5 & 86.60 & 80.23 & 1.65 \\
\hline 6 & 70.20 & 91.43 & 19.10 \\
\hline 7 & 88.20 & 0.00 & 0.00 \\
\hline 8 & 78.07 & 6.60 & 6.50 \\
\hline 9 & 92.87 & 4.87 & 55.50 \\
\hline 10 & 25.87 & 0.00 & 0.00 \\
\hline 11 & 46.67 & 23.57 & 10.25 \\
\hline 12 & 94.73 & 6.60 & 51.00 \\
\hline 13 & 5.40 & 35.97 & 5.45 \\
\hline 14 & 85.90 & 44.20 & 58.55 \\
\hline 15 & 95.23 & 0.00 & 57.30 \\
\hline 16 & 19.40 & 0.00 & 2.65 \\
\hline 17 & 17.77 & 14.07 & 7.05 \\
\hline 18 & 95.70 & 66.23 & 27.70 \\
\hline 19 & 88.83 & 93.40 & 2.40 \\
\hline 20 & 73.80 & 0.00 & 17.45 \\
\hline 21 & 8.97 & 42.70 & 8.70 \\
\hline 22 & 9.39 & 1.50 & 2.65 \\
\hline 23 & 95.27 & 11.53 & 23.30 \\
\hline 24 & 85.33 & 15.33 & 78.90 \\
\hline 25 & 94.70 & 9.70 & 75.10 \\
\hline 26 & 24.67 & 0.00 & 24.40 \\
\hline 27 & 86.63 & 9.67 & 47.05 \\
\hline 28 & 59.43 & 0.00 & 17.05 \\
\hline 29 & 44.57 & 6.53 & 16.05 \\
\hline 30 & 3.37 & 11.97 & 1.45 \\
\hline 31 & 19.10 & 11.03 & 4.60 \\
\hline
\end{tabular}




\begin{tabular}{|c|c|c|c|}
\hline \multirow{2}{*}{ S. No. } & \multicolumn{3}{|c|}{ DMI\% } \\
\hline & Sg445 & Sg519 & Sg526 \\
\hline 32 & 93.20 & 0.00 & 33.90 \\
\hline 33 & 53.03 & 0.00 & 4.45 \\
\hline 34 & 76.57 & 5.97 & 18.80 \\
\hline 35 & 94.37 & 0.00 & 55.20 \\
\hline 36 & 88.87 & 15.13 & 33.30 \\
\hline 37 & 98.40 & 51.27 & 0.00 \\
\hline 38 & 26.80 & 6.90 & 11.45 \\
\hline 39 & 65.10 & 0.00 & 5.15 \\
\hline 40 & 97.63 & 100.00 & 15.00 \\
\hline 41 & 96.17 & 2.77 & 0.00 \\
\hline 42 & 6.00 & 0.00 & 1.85 \\
\hline 43 & 35.73 & 50.00 & 0.00 \\
\hline 44 & 92.60 & 7.13 & 38.70 \\
\hline 45 & 57.83 & 12.60 & 1.40 \\
\hline 46 & 60.80 & 86.63 & 28.60 \\
\hline 47 & 97.93 & 8.93 & 30.85 \\
\hline 48 & 60.87 & 13.20 & 22.00 \\
\hline 49 & 39.50 & 24.27 & 0.00 \\
\hline 50 & 98.03 & 100.00 & 6.75 \\
\hline 51 & 24.10 & 25.17 & 0.00 \\
\hline 52 & 17.67 & 8.10 & 2.25 \\
\hline 53 & 5.30 & 1.13 & 0.00 \\
\hline 54 & 87.83 & 60.23 & 24.75 \\
\hline 55 & 92.47 & 50.33 & 13.70 \\
\hline 56 & 55.17 & 66.67 & 6.25 \\
\hline 57 & 13.40 & 26.50 & 2.00 \\
\hline 58 & 48.43 & 90.90 & 15.25 \\
\hline 59 & 43.50 & 0.00 & 1.45 \\
\hline 60 & 85.23 & 100.00 & 3.75 \\
\hline 61 & 0.00 & 0.00 & 1.45 \\
\hline 62 & 97.93 & 98.23 & 21.55 \\
\hline 63 & 83.90 & 14.47 & 34.50 \\
\hline 64 & 87.10 & 1.13 & 21.75 \\
\hline 65 & 12.73 & 0.00 & 3.00 \\
\hline 66 & 82.77 & 12.27 & 3.35 \\
\hline 67 & 82.60 & 0.00 & 0.00 \\
\hline 68 & 96.30 & 1.20 & 98.65 \\
\hline 69 & 0.00 & 1.20 & 0.00 \\
\hline 70 & 66.13 & 2.57 & 16.05 \\
\hline
\end{tabular}




\begin{tabular}{|c|c|c|c|}
\hline \multirow{2}{*}{ S. No. } & \multicolumn{3}{|c|}{ DMI\% } \\
\hline & Sg445 & Sg519 & Sg526 \\
\hline 71 & 9.37 & 14.63 & 8.30 \\
\hline 72 & 87.73 & 75.63 & 33.30 \\
\hline 73 & 92.47 & 91.67 & 17.90 \\
\hline 74 & 3.20 & 1.50 & 2.25 \\
\hline 75 & 3.27 & 0.00 & 7.40 \\
\hline 76 & 97.53 & 98.67 & 7.95 \\
\hline 77 & 76.73 & 13.40 & 89.90 \\
\hline 78 & 95.47 & 100.00 & 8.60 \\
\hline 79 & 44.47 & 31.20 & 5.95 \\
\hline 80 & 81.60 & 13.07 & 17.00 \\
\hline 81 & 63.47 & 3.03 & 8.85 \\
\hline 82 & 14.70 & 0.00 & 89.55 \\
\hline 83 & 66.67 & 25.23 & 42.90 \\
\hline 84 & 28.60 & 53.97 & 2.80 \\
\hline 85 & 67.57 & 97.90 & 6.25 \\
\hline 86 & 3.83 & 17.40 & 3.75 \\
\hline 87 & 66.63 & 4.57 & 19.40 \\
\hline 88 & 37.20 & 0.00 & 2.25 \\
\hline 89 & 75.07 & 20.83 & 9.35 \\
\hline 90 & 91.33 & 1.40 & 63.95 \\
\hline 91 & 100.00 & 98.23 & 9.25 \\
\hline 92 & 100.00 & 43.80 & 77.10 \\
\hline 93 & 45.83 & 31.13 & 12.10 \\
\hline 94 & 60.47 & 1.07 & 31.40 \\
\hline 95 & 24.30 & 0.00 & 19.95 \\
\hline 96 & 75.63 & 13.63 & 45.65 \\
\hline 97 & 1.60 & 27.73 & 1.70 \\
\hline 98 & 87.90 & 1.07 & 8.40 \\
\hline 99 & 16.90 & 1.20 & 10.85 \\
\hline 100 & 74.43 & 2.47 & 11.05 \\
\hline 101 & 78.33 & 47.50 & 9.45 \\
\hline 102 & 96.13 & 0.00 & 9.75 \\
\hline 103 & 93.07 & 5.40 & 100.00 \\
\hline 104 & 34.73 & 0.00 & 3.35 \\
\hline 105 & 65.90 & 0.00 & 9.25 \\
\hline 106 & 25.00 & 10.07 & 100.00 \\
\hline 107 & 95.70 & 10.73 & 100.00 \\
\hline 108 & 71.10 & 25.57 & 59.80 \\
\hline 109 & 28.33 & 0.00 & 33.30 \\
\hline
\end{tabular}




\begin{tabular}{|c|c|c|c|}
\hline \multirow{2}{*}{ S. No. } & \multicolumn{3}{|c|}{ DMI\% } \\
\hline & Sg445 & Sg519 & Sg526 \\
\hline 110 & 32.73 & 0.00 & 45.50 \\
\hline 111 & 64.87 & 8.63 & 3.55 \\
\hline 112 & 84.50 & 4.47 & 3.15 \\
\hline 113 & 65.80 & 22.40 & 31.95 \\
\hline 114 & 98.13 & 12.63 & 9.70 \\
\hline 115 & 64.37 & 88.93 & 6.40 \\
\hline 116 & 90.40 & 24.67 & 40.50 \\
\hline 117 & 37.80 & 14.03 & 5.55 \\
\hline 118 & 72.60 & 53.73 & 63.40 \\
\hline 119 & 71.93 & 29.43 & 2.80 \\
\hline 120 & 97.57 & 2.23 & 87.45 \\
\hline 121 & 31.80 & 69.07 & 0.00 \\
\hline 122 & 94.90 & 100.00 & 11.90 \\
\hline 123 & 34.90 & 23.40 & 1.45 \\
\hline 124 & 97.83 & 1.07 & 3.05 \\
\hline 125 & 33.30 & 0.00 & 22.45 \\
\hline 126 & 91.83 & 17.77 & 14.35 \\
\hline 127 & 100.00 & 17.80 & 7.15 \\
\hline 128 & 40.63 & 80.53 & 0.00 \\
\hline 129 & 82.43 & 18.67 & 7.55 \\
\hline 130 & 86.23 & 20.13 & 17.10 \\
\hline 131 & 91.17 & 26.33 & 23.95 \\
\hline 132 & 45.70 & 22.33 & 1.65 \\
\hline 133 & 79.53 & 0.00 & 0.00 \\
\hline 134 & 89.83 & 0.00 & 86.55 \\
\hline 135 & 100.00 & 46.67 & 67.95 \\
\hline 136 & 91.87 & 27.53 & 90.90 \\
\hline 137 & 33.80 & 21.53 & 2.50 \\
\hline 138 & 83.83 & 0.00 & 54.25 \\
\hline 139 & 28.20 & 0.00 & 13.40 \\
\hline 140 & 28.93 & 42.90 & 21.85 \\
\hline 141 & 12.43 & 0.00 & 1.85 \\
\hline 142 & 74.17 & 73.63 & 59.40 \\
\hline 143 & 86.30 & 1.67 & 10.30 \\
\hline 144 & 23.77 & 22.77 & 1.65 \\
\hline 145 & 81.43 & 64.73 & 87.35 \\
\hline 146 & 98.03 & 10.87 & 83.20 \\
\hline 147 & 75.10 & 100.00 & 5.65 \\
\hline 148 & 74.81 & 71.45 & 23.60 \\
\hline
\end{tabular}




\begin{tabular}{|c|c|c|c|}
\hline \multirow{2}{*}{ S. No. } & \multicolumn{3}{|c|}{ DMI\% } \\
\hline & Sg445 & Sg519 & Sg526 \\
\hline 149 & 92.90 & 0.00 & 25.50 \\
\hline 150 & 82.93 & 26.83 & 14.75 \\
\hline 151 & 34.80 & 2.43 & 9.40 \\
\hline 152 & 96.87 & 6.07 & 24.45 \\
\hline 153 & 97.63 & 10.13 & 34.85 \\
\hline 154 & 81.93 & 0.00 & 5.80 \\
\hline 155 & 95.90 & 3.07 & 93.35 \\
\hline 156 & 9.90 & 12.63 & 0.00 \\
\hline 157 & 16.97 & 0.00 & 0.00 \\
\hline 158 & 13.83 & 0.00 & 0.00 \\
\hline 159 & 16.27 & 2.37 & 7.50 \\
\hline 160 & 21.07 & 10.17 & 0.00 \\
\hline 161 & 36.63 & 11.03 & 0.00 \\
\hline 162 & 33.27 & 9.10 & 0.00 \\
\hline 163 & 75.33 & 11.97 & 5.30 \\
\hline 164 & 95.77 & 4.20 & 88.95 \\
\hline 165 & 75.07 & 39.43 & 30.95 \\
\hline 166 & 94.43 & 2.37 & 87.50 \\
\hline 167 & 53.90 & 96.43 & 33.85 \\
\hline 168 & 89.93 & 2.10 & 47.70 \\
\hline 169 & 89.00 & 61.70 & 6.30 \\
\hline 170 & 25.70 & 4.10 & 23.00 \\
\hline 171 & 100.00 & 0.00 & 0.00 \\
\hline 172 & 95.47 & 15.40 & 4.05 \\
\hline 173 & 74.83 & 9.43 & 28.25 \\
\hline 174 & 100.00 & 8.57 & 98.10 \\
\hline 175 & 15.87 & 0.00 & 0.00 \\
\hline 176 & 58.73 & 1.67 & 0.00 \\
\hline 177 & 45.77 & 28.77 & 2.85 \\
\hline 178 & 48.50 & 6.03 & 1.30 \\
\hline 179 & 98.67 & 98.57 & 43.75 \\
\hline 180 & 97.43 & 6.80 & 24.50 \\
\hline 181 & 84.97 & 0.00 & 0.00 \\
\hline 182 & 70.20 & 8.97 & 4.40 \\
\hline 183 & 92.40 & 7.83 & 62.75 \\
\hline 184 & 44.20 & 14.03 & 7.00 \\
\hline 185 & 95.23 & 94.87 & 25.65 \\
\hline 186 & 96.80 & 96.63 & 0.00 \\
\hline 187 & 66.87 & 64.63 & 0.00 \\
\hline
\end{tabular}




\begin{tabular}{|c|c|c|c|}
\hline \multirow{2}{*}{ S. No. } & \multicolumn{3}{|c|}{ DMI\% } \\
\hline & Sg445 & Sg519 & Sg526 \\
\hline 188 & 57.93 & 77.27 & 0.00 \\
\hline 189 & 0.00 & 0.00 & 0.00 \\
\hline 190 & 93.50 & 1.67 & 8.35 \\
\hline 191 & 39.13 & 15.47 & 5.25 \\
\hline 192 & 64.07 & 9.10 & 11.50 \\
\hline 193 & 12.03 & 64.03 & 4.95 \\
\hline 194 & 50.87 & 72.23 & 22.45 \\
\hline 195 & 100.00 & 65.10 & 0.00 \\
\hline 196 & 62.73 & 0.00 & 11.25 \\
\hline 197 & 98.23 & 45.83 & 57.40 \\
\hline 198 & 86.53 & 0.00 & 21.30 \\
\hline 199 & 88.03 & 77.23 & 0.00 \\
\hline 200 & 60.13 & 23.50 & 25.45 \\
\hline 201 & 71.10 & 21.90 & 11.00 \\
\hline 202 & 34.17 & 83.00 & 5.15 \\
\hline 203 & 92.07 & 0.00 & 91.30 \\
\hline 204 & 62.03 & 25.27 & 51.90 \\
\hline 205 & 86.67 & 100.00 & 51.65 \\
\hline 206 & 10.90 & 33.47 & 4.90 \\
\hline 207 & 3.17 & 57.07 & 0.00 \\
\hline 208 & 95.83 & 0.00 & 42.95 \\
\hline 209 & 17.07 & 0.00 & 0.00 \\
\hline 210 & 84.90 & 62.23 & 0.00 \\
\hline 211 & 71.00 & 0.00 & 0.00 \\
\hline 212 & 28.27 & 1.27 & 1.90 \\
\hline 213 & 54.77 & 0.00 & 95.85 \\
\hline 214 & 55.33 & 4.43 & 14.25 \\
\hline 215 & 39.27 & 0.00 & 4.75 \\
\hline 216 & 25.73 & 1.87 & 0.00 \\
\hline 217 & 87.60 & 2.10 & 1.50 \\
\hline 218 & 95.27 & 11.67 & 16.45 \\
\hline 219 & 4.30 & 12.17 & 0.00 \\
\hline 220 & 33.30 & 0.00 & 8.15 \\
\hline 221 & 86.77 & 63.57 & 27.20 \\
\hline 222 & 99.93 & 42.20 & 22.50 \\
\hline 223 & 97.43 & 9.50 & 12.90 \\
\hline 224 & 5.33 & 11.47 & 0.00 \\
\hline 225 & 100.00 & 81.53 & 15.00 \\
\hline 226 & 85.20 & 31.77 & 30.80 \\
\hline
\end{tabular}




\begin{tabular}{|c|c|c|c|}
\hline \multirow{2}{*}{ S. No. } & \multicolumn{3}{|c|}{ DMI\% } \\
\hline & Sg445 & Sg519 & Sg526 \\
\hline 227 & 86.30 & 51.33 & 38.65 \\
\hline 228 & 1.60 & 3.37 & 4.05 \\
\hline 229 & 74.23 & 2.93 & 93.65 \\
\hline 230 & 6.40 & 0.00 & 8.70 \\
\hline 231 & 25.10 & 2.17 & 0.00 \\
\hline 232 & 26.23 & 15.60 & 4.95 \\
\hline 233 & 97.43 & 45.57 & 100.00 \\
\hline 234 & 72.07 & 100.00 & 45.30 \\
\hline 235 & 95.13 & 11.33 & 7.40 \\
\hline 236 & 84.53 & 8.97 & 44.25 \\
\hline 237 & 7.83 & 0.00 & 0.00 \\
\hline 238 & 100.00 & 100.00 & 30.90 \\
\hline 239 & 100.00 & 1.60 & 63.95 \\
\hline 240 & 85.37 & 100.00 & 16.20 \\
\hline 241 & 70.27 & 0.00 & 24.50 \\
\hline 242 & 91.30 & 12.77 & 10.55 \\
\hline 243 & 98.60 & 4.20 & 76.40 \\
\hline 244 & 98.13 & 3.03 & 24.40 \\
\hline 245 & 29.97 & 12.80 & 9.35 \\
\hline 246 & 92.23 & 0.00 & 5.15 \\
\hline 247 & 92.07 & 1.27 & 21.15 \\
\hline 248 & 98.73 & 28.03 & 98.20 \\
\hline 249 & 14.73 & 2.57 & 10.10 \\
\hline 250 & 61.70 & 10.33 & 34.05 \\
\hline 251 & 48.03 & 69.30 & 7.70 \\
\hline 252 & 100.00 & 3.33 & 70.85 \\
\hline 253 & 33.87 & 0.00 & 8.40 \\
\hline 254 & 82.57 & 91.40 & 6.80 \\
\hline 255 & 55.47 & 32.80 & 51.10 \\
\hline 256 & 75.63 & 6.33 & 24.40 \\
\hline 257 & 95.70 & 10.57 & 39.25 \\
\hline 258 & 13.00 & 39.73 & 10.90 \\
\hline 259 & 92.03 & 7.20 & 47.90 \\
\hline 260 & 47.37 & 4.83 & 2.65 \\
\hline 261 & 94.93 & 0.00 & 10.80 \\
\hline 262 & 24.10 & 55.23 & 52.25 \\
\hline 263 & 93.87 & 0.00 & 55.25 \\
\hline 264 & 0.00 & 0.00 & 13.70 \\
\hline 265 & 55.03 & 0.00 & 23.90 \\
\hline
\end{tabular}




\begin{tabular}{|c|c|c|c|}
\hline \multirow{2}{*}{ S. No. } & \multicolumn{3}{|c|}{ DMI\% } \\
\hline & Sg445 & Sg519 & Sg526 \\
\hline 266 & 14.00 & 0.00 & 14.30 \\
\hline 267 & 69.67 & 0.00 & 96.75 \\
\hline 268 & 95.30 & 2.30 & 41.75 \\
\hline 269 & 100.00 & 64.07 & 51.30 \\
\hline 270 & 73.67 & 70.67 & 35.70 \\
\hline 271 & 53.80 & 55.63 & 2.50 \\
\hline 272 & 97.93 & 24.53 & 3.15 \\
\hline 273 & 91.40 & 98.23 & 5.60 \\
\hline 274 & 100.00 & 100.00 & 30.00 \\
\hline 275 & 98.77 & 37.70 & 14.25 \\
\hline 276 & 15.40 & 0.00 & 0.00 \\
\hline 277 & 2.43 & 13.07 & 2.65 \\
\hline 278 & 100.00 & 24.07 & 87.80 \\
\hline 279 & 65.20 & 25.57 & 13.80 \\
\hline 280 & 72.63 & 26.07 & 0.00 \\
\hline 281 & 5.87 & 0.00 & 0.00 \\
\hline 282 & 23.90 & 1.87 & 0.00 \\
\hline 283 & 3.70 & 5.17 & 34.90 \\
\hline 284 & 100.00 & 14.40 & 68.35 \\
\hline 285 & 89.67 & 14.17 & 18.35 \\
\hline 286 & 70.67 & 93.43 & 30.50 \\
\hline 287 & 74.67 & 31.70 & 54.40 \\
\hline 288 & 74.17 & 0.00 & 8.35 \\
\hline 289 & 98.23 & 93.77 & 25.65 \\
\hline 290 & 96.73 & 36.63 & 100.00 \\
\hline 291 & 5.13 & 0.00 & 2.65 \\
\hline 292 & 62.33 & 66.40 & 18.35 \\
\hline 293 & 22.80 & 3.47 & 2.95 \\
\hline 294 & 68.98 & 14.27 & 13.80 \\
\hline 295 & 74.03 & 5.67 & 20.60 \\
\hline P1 & 100 & 100 & 91.15 \\
\hline P2 & 3.0 & 3.2 & 2.0 \\
\hline $7042(S)$ & 100 & 100 & 94.4 \\
\hline IP18292 & 64.46 & 100 & 71.9 \\
\hline Mean & 62.64 & 26.19 & 24.38 \\
\hline SEm+/- & 4.70 & 2.82 & 1.68 \\
\hline SEd+/- & 6.66 & 3.99 & 2.39 \\
\hline $\operatorname{CV}(\%)$ & 13.023 & 18.68 & 9.81 \\
\hline $\begin{array}{c}\text { Heritability } \\
\text { (broad sense) }\end{array}$ & 0.93 & 0.97 & 0.99 \\
\hline
\end{tabular}


Host plant resistance is the most economic and efficient strategy for the management of downy mildew of pearl millet. Effective resistance breeding programmes require close monitoring of virulence change in the pathogen and identification of new resistance sources to the new virulent strains. Virulence change in $S$. graminicola populations is monitored through a collaborative pearl millet downy mildew nursery, on-farm surveys for downy mildew incidence and by characterizing pathogen isolates collected from highly susceptible cultivars in the farmers' fields on a set of putative differential hosts (Sivaramakrishnan et al., 2003; Thakur et al., 2004).

Breeding crop varieties with durable resistance to diseases is made difficult by the variability in the pathogen populations (Christ et al., 1987; Leonard, 1977). Genetic resistance in a cultivar at one location may not function at another location because of the differences in virulence in the pathogen populations (Flor, 1971; Kulkarni and Chopra, 1982; Vanderplank, 1984). Differential host varieties are useful in the analysis of pathogen variability at various locations on the basis of clearly visible resistant and susceptible reactions (Vanderplank, 1984; Wolfe and Knott, 1982). According to Flors (Flor, 1971) gene-for-gene hypothesis, a series of inbred lines with distinct resistance genes will differentiate discrete races of the pathogen. Theoretically, pathogen populations are composed of numerous strains, each of which exists at certain frequency that describes the probability of encounter (Gale, 1987).

Genetic management of downy mildew in pearl millet could be strategically planned on a regular basis, because of a highly dynamic $S$. graminicola-pearl millet system. The International Pearl Millet Downy Mildew Nursery (IPMDMN) conducted to test the stability of resistance also provided evidence of variable pathogen populations in countries in Africa and India (Singh, 1995; Singh et al., 1993).

The control entries 7042(S) showed 94.4$100 \%$ DMI and IP 18292 was found to possess 64.46 - 100\% DMI across all Indian isolates of pathogen.

The results of this study indicate the importance of identifying Downy Mildew Incidence for an effective downy mildew resistance breeding in pearl millet.

The results of this study can be used to mapping of DMR QTLs and this study also indicating the variability in the virulence level of pathogen isolates for the same genotypes, which aggravating the necessity of hostpahotype specific resistance breeding.

\section{Acknowledgements}

The first and third authors thank Acharya N.G. Ranga Agricultural University (ANGRAU) for financial help in the form of stipend during the course of work.

\section{References}

Christ, B.J., C.O. Person and D.D. Pope 1987. The genetic determination of variation in pathogenicity. In: Wolfe, M.S., Caten, C.E. (Eds.), Populations of Plant Pathogens: Their Dynamics and Genetics. Blackwell Scientific, Oxford, UK, pp. 7-19.

Flor, H.H., 1971. Current status of the genefor-gene concept. Annual Review of Phytopathology 9:275-296.

Gale, J.S., 1987. Factors delaying the spread of a virulent mutant of a fungal pathogen: some suggestions from population genetics. In: Wolfe, M.S., Caten, C.E. (eds.), Populations of the 
Plant Pathogens: Their Dynamics and Genetics. Blackwell Scientific, Oxford, UK, pp. 55-62.

Kulkarni, R.N., and V.L. Chopra 1982. Environment as the cause of differential interaction between host cultivars and pathogenic races. Letter to the Editor, Phytopathology 72: 1384-1386.

Leonard, K.J., 1977. Selection pressures and plant pathogens. Ann. N.Y. Acad. Sci. 287: 207-222.

Pushpavathi, B., R.P. Thakur and K.C. Rao 2006a. Fertility and mating type frequency in Indian isolates of Sclerospora graminicola, the downy mildew pathogen of pearl millet. Plant Disease 90: 211-214.

Pushpavathi, B., R.P. Thakur, K.C. Rao and V.P. Rao 2006b. Characterization of Sclerospora graminicola isolates from pearl millet for virulence and genetic diversity. Plant Pathology Journal 22: 28-35.

Rajaram, V., T. Nepolean, S. Senthilvel, R.K. Varshney, V. Vadez, R.K. Srivastava, T.M. Shah, A. Supriya, S. Kumar, B.R. Kumari, A. Bhanuprakash, M.L. Narasu, O.R. Lizarazu and C.T. Hash 2013. Pearl millet consensus linkage map constructed using four RIL mapping populations and newly developed EST-SSRs. BMC Genomics 14: 159

Rao, V.P., R.P. Thakur, K.N. Rai and Y.K. Sharma 2005. Downy mildew incidence on pearl millet cultivars and pathogenic variability among isolates of Sclerospora graminicola in Rajasthan. International Sorghum and Millets Newsletter 46: 107-110.

Sastry, J.G., S. Sivaramakrishnan, V.P. Rao, R.P. Thakur, R.S. Singru, V.S. Gupta and P.K. Ranjekar 2001. Genetic basis of host specificity in Sclerospora graminicola, the pearl millet downy mildew pathogen. Indian
Phytopathology 54: 323-328.

Singh, S.D., 1995. Downy mildew of pearl millet. Plant Disease 79: 545-550.

Singh, S.D., R. Gopinath and M.N. Pawar 1987. Effects of environmental factors in asexual sporulation of Sclerospora graminicola. Indian Phytopathology 40: 186-193.

Singh, S.D., S.B. King and J. Werder 1993. Downy mildew disease of pearl millet. Information Bulletin No. 37. International Crops Research Institute for the Semi-Arid Tropics. Patancheru, A.P. 502 324, India.

Sivaramakrishnan, S., R.P. Thakur, S. Kannan and V.P. Rao 2003. Pathogenic and genetic diversity among Indian isolates of Sclerospora graminicola from pearl millet. Indian Phytopathology 56: 392 397.

Thakur R.P., K.G. Shetty and S.B. King 1992. Selection for host-specific virulence in asexual populations of Sclerospora graminicola. Plant Pathology 41: 626632.

Thakur, R.P. 1999. Pathogen diversity and plant disease management. Indian Phytopathology 52: 1-9.

Thakur, R.P., and V.P. Rao 1993. Intrapopulation genetic diversity for virulence and aggressiveness in Sclerospora graminicola. The Sixth International Congress of Plant Pathology. Montreal. 166.

Thakur, R.P., H.S. Shetty and I.S. Khairwal 2006. Pearl millet downy mildew research in India: progress and perspectives. International Sorghum and Millets Newsletter 47: 125-130.

Thakur, R.P., K.N. Rai, I.S. Khairwal and R.S. Mahala 2008. Strategy for downy mildew resistance breeding in pearl millet in India. SAT eJournal 6: 1-11.

Thakur, R.P., S. Rajan and V.P. Rao 2011. Screening techniques for pearl millet diseases. Information Bulletin No. 89. 
Patancheru, Andhra Pradesh, India: International Crops Research Institute for the Semi-Arid Tropics. 56.

Thakur, R.P., V.P. Rao, H.S.A. Shetty and V.V. Datar 2003. Field surveys of pearl millet downy mildew: effects of hybrids, fungicide and cropping sequence. Journal of Mycology and Plant Pathology 33: 387-394.

Thakur, R.P., V.P. Rao, B.M. Wu, K.V. Subbarao, H.S. Shetty, G. Singh, C. Lukose, M.S. Panwar, P. Sereme and D.E. Hess et al., 2004. Host resistance stability to downy mildew in pearl millet and pathogenic variability in Sclerospora graminicola. Crop Protection 23: 901-908.

Vanderplank, J.E., 1984. Disease Resistance in Plants, 2nd Edition. Academic Press, Orlando.

Wolfe, M.S., and D.R. Knott 1982. Population of plant pathogens: some constraints on analysis of variation in pathogenicity. Plant Pathology 31: 7990.

\section{How to cite this article:}

Anishetti Saritha, Rakesh K. Srivastava, Chelpuri Durgaraju, Narayan Reddy, Rajan Sharma, Pooja Katiyar and Kuldeep Singh Dangi. 2017. Genetic Variability for Downy Mildew Disease Incidence against Virulent Downy Mildew Isolates in Mapping Population of Pearl Millet. Int.J.Curr.Microbiol.App.Sci. 6(10): 595-608. doi: https://doi.org/10.20546/ijcmas.2017.610.073 